\title{
Review on Epidemiology, Diagnosis Control and Prevention of Brucellosis
}

\author{
Tesfahun Demeke Dana* \\ Wolaita Sodo University School of Veterinary Medicine
}

*Corresponding Author: Tesfahun Demeke Dana, Wolaita Sodo University School of Veterinary Medicine

\begin{abstract}
Bovine brucellosis is a worldwide zoonotic disease of animals and humans which result socioeconomic impacts on the worldwide. It is caused by various species of genus Brucella. Primarily brucellosis is disease of animals however human also susceptible for the disease which result more than 500,000 cases of human per year all around the world. The disease is transmitted by ingestion of Brucella induced feed, consumption of dairy products from infected animals, close contact with infected animal as well as respiratory aerosols between animals. Human acquire the disease by consumption of raw dairy products, close contact with infected animals and through respiratory rout. In animals the clinical signs are related to reproductive systems. In pregnant cows it cause Retained placenta, metritis and the most common sign in Brucella infected cow is Abortions, which is occur after the fifth month of gestation. In bulls it is characterized by Orchitis, epididymitis and Vasculitis. Various methods are employed for the diagnosis of brucellosis including rose Bengal test, complement fixation test (CFT), bacterial culture and milk ring test. Among Serological tests, rose Bengal tests are used to screen animals however CFT is used to confirm the causative agent. The bacillus can be isolated by bacterial culture on Brucella selective media. In developed countries Brucellosis was successfully eradicated by using test and slaughter method of control. However this method of control is not employed in developing countries because of economic aspect of the countries. In developing countries other methods such as immunization of animals, farm sanitation and community awareness were applied to reduce the spread of disease.
\end{abstract}

Keywords: brucellosis, genus Brucella, infected animal, zoonosis

\section{INTRODUCTION}

Brucellosis is one of the most prevalent bacterial zoonotic diseases causing significant economic losses due to the livestock abortion, and also it is possibly a life-threatening multi-system disease in human. [1] It is caused by several species of the genus Brucella. This genus encompasses ten known species, containing three species which are common, and those species has economic and public health significance. These species are B. abortus, B. melitensis and B. suise. Susceptibility to brucellosis varies among individual animals. It depends on the animals' natural resistance, age, sex, level of immunity and environmental stress. [2,3]

The first person who isolated Brucella is David Bruce. He isolated B. melitensis from a liver of British soldier who dead by Malta fever in 1887. For almost 20 years aier isolation of M. melitensis, Malta fever remained a mystery and was thought to be a vector-borne disease until Hemistocles Zammit accidentally demonstrated the zoonotic nature of the disease in 1905 by isolating B. melitensis from goat's milk. Goats were not believed to be a source of disease before the bacteria isolated from goat's milk, since they did not become ill when inoculated with Brucella cultures. Hemistocles Zammit's discovery shows that the disease could be found in healthy goats and they can be a carrier for the disease. This discovery has been called as one of the greatest progresses ever made in the study of epidemiology. $[4,5]$

Infected cattles are the main source of the infection. They shed the agent via milk, vaginal discharge, aborted fetus and placenta. Cattles usually acquire the disease through in contact with infected birth products and consumption of milk from infected cow. [6] Although brucellosis is primarily the disease of cattles, human being is also susceptible to the disease. Human often acquire the disease by ingestion of raw dairy products and undercooked meat products, close contact, inhalation or accidental inoculation. [7] 
Diagnosis of the disease depends on isolation and identification of Brucella from aborted materials, udder secretions or from tissues removed at post-mortem or patient's serum by detection of specific antibodies using appropriate serological methods. Presumptive diagnosis can be made by assessing specific cell mediated or serological responses to Brucella antigens. [8]

Although the nature of brucellosis makes it very difficult to treat, the longer treatment with antibiotics results the longer the chance of recovery. Some studies shows that the combination of antibiotics may result best way to the cure the disease. [9] Brucellosis can be considered a paradigm of the need for a "One World, One Health" strategy given that the only approach to achieve the control and subsequent eradication of this zoonotic disease is the cooperation between the industry, producers, and public and animal health authorities. [10]

Generally, there are three main strategies for control and prevention of the disease: the first one is Test and slaughter which had been performed in developed countries, strict bio security at the farm level and Immunization of the susceptible population. [11] The Importance of such assessments makes it easy to understand the nature of the disease, so the objective of this review is

- to assess the epidemiology and diagnosis of brucellosis

- to evaluate the public health importance of the disease

- to highlight the control and prevention methods of the disease

\subsection{Etiology and Characteristics of Brucellosis}

Brucellosis is an infectious disease of animals and humans which is caused by gram negative bacilli belongs to the genus Brucella. This genus encompasses ten known species, containing three species which are common, and those species has economic and public health significance. These species are B. abortus which is the main causative agent of brucellosis in cattles, B. melitensis which cause brucellosis in sheep and goat and B. suise which affects swine. Those Brucella species except B. suis biovar has public health significance. In addition to these species, recently two species called B. cetaceae and B. pinnipediae were added to genus Brucella. This species were isolated from marine mammals, cetaceans, and pinnipeds. [3, 12, 13] The bacterium is live as, non-motile facultative intracellular pathogens of the reticuloendothelial cells of terrestrial and marine mammal hosts. The bacterium is of $0.5-0.7 \mu$ in diameter and $0.6-1.5 \mu$ in length. They are oxidase, catalase and urease positive. The genome of Brucella lack plasmid but it has circular chromosomes this indicates that the presence of significant difference when it is compared to the single chromosome of many bacteria. The mechanisms of their virulence and survival inside professional phagocytes partially remains an enigma as Brucella has not been shown to produce the virulence factors, such as cytolysins, capsules, exotoxins, secreted proteases, pili and /or fimbriae, flagella, phage encoded toxins and virulence plasmids, implicated in such processes in other bacteria. $[14,15,16,17]$

\subsection{Transmission}

Cow which is infected by brucellosis is the main source of the infection. The bacilli from the infected cow are discharged through several ways including through aborted fetus, uterine discharge, placenta as well as milk. Cattles often acquire the disease through ingestion of Brucella induced feed; contact to vaginal discharge of infected animal, contact to birth products and uterine discharge. The agent can be found in the discharge of uterine beginning before 2 week of abortion or parturition, and lasting 2 to 3 week thereafter. The period between the infection of an animal by the virus and showing the clinical signs is quite variable, ranging from 2 weeks to 1 year or longer. [18]

In many cases, once animals are infected by the disease, they will remain in the disease for years or remain infected for rest of their life. [6] Humans often acquire the disease by consumption of dairy products from infected animals and by close contact with infected animals. [19]

\section{Clinical SignS}

The period between the infection of an animal by the virus and showing the clinical signs is quite variable, ranging from 2 weeks to 1 year or longer. [18] Except cat, all species of animals are susceptible for brucellosis; cats are naturally resistant for infection of Brucella. Brucellosis can be tentatively diagnosed by clinical signs. Most clinical signs are related to reproductive systems. In pregnant cows it cause Retained placenta, metritis and the most common sign in Brucella infected 
cow is Abortions, which is occur after the fifth month of gestation. When a pregnant animal is infected by Brucella, a visible swelling of the mammary gland to the navel region and bleeding from the vagina is not uncommon, even if the cow does not abort. In bulls it is characterized by causing inflammation of genital organs such as inflammation of testicle, epididymis and vas deference. In addition to inflammation of genital organ, fever is also the main clinical symptom in bulls. In bulls, when it reaches sever level it may cause testicular abscess and Orchitis that results permanent infertility. [18, 20, 21, 22]

In human, the clinical presentation of brucellosis is non-specific and requires laboratory testing for confirmation. Common presentations include fever, nausea, anorexia, headache, sweating, and prostration and these symptoms can be coupled with gastro-intestinal, musculoskeletal, hepato-biliary and meningeo-vascular complications. [23]

\subsection{Pathogenesis}

The organism can enter in to animal via multiple routes. Among those rout the most common way is GI tract in which after ingestion of the agent, it can get accesses to enter in to circulation via gastrointestinal tract. Although GI tract is most common rout, inhalation is also possible way to get in to the body. Once the bacterium enters to the body then the bacilli can translocate to lymphatic system then it gets access to enter into circulation and result bacteremia. Organs that support growth of a bacterium include pregnant uteri, male genital organs, mammary glands, and associated supramammary lymph nodes. [24]

The ponderous lodgement of B. abortus in the placenta is resulted from intracellular multiplication of the bacilli with in chorioallantoic trophoblast. The growth of Brucella organisms in trophoblasts may be enhanced by their erythritol and/or hormone content, because both erythritol and progesterone augment the growth of B. abortus in vitro. [18]

\section{DIAGNOSIS OF BRUCELLOSIS}

\subsection{Bacteriological tests}

\subsubsection{Stained Smears}

Specimen used for identification of the bacilli include aborted fetus stomach, placental cotyledon, spleen, liver, and uterine discharge. This specimen taken can be smeared on slide and stained by using either modified staining method is called Ziehl-Neelsen or Kosters' methods. After staining it is examined under microscope, the presence of small red colored, weakly acid-fast organism, coccobacilli in clumps with Brucella morphology is presumptive evidence of brucellosis. The investigation should be done carefully because of other intracellular organism such as Coxiella burnetii or Chlamydia resembles Brucella. [3, 25]

\subsection{Bacteriological Cultures}

The "gold standard" in the diagnosis of brucellosis is bacterial isolation, which requires long cultivation periods and is often unsuccessful. [26] Usually all strain of Brucella are growing slow in culture media. The specimen collected for culture of Brucella can be either blood or bone marrow sample and it can be cultured in 5\% blood agar media. Most of time the specimen is contaminated with bacteria and fungus, to be sure it can be cultured on Brucella selective media such as Farrell's medium. The agent is incubated in Farrell's media for 72 hour, however if it is not grow within a week it can be decided as a negative result. [25, 27]

In addition to Farrell's medium there are a range of commercially available culture media for growing Brucella. The most common basal media in use are: Triptcase soy (BBL®), Bacto Tryptose (Difco®), Triptic soy (Gibco®), Tryptone soya (Oxoid®). [28]

\subsection{Serological Tests}

Serological tests are the most important diagnostic tests for screening test of brucellosis, that it plays crucial role in the control and eradication program of brucellosis. Attenuated bacteria or purified fragment such as lipopolysaccharide or membrane proteins is used in the detection of antibodies which is produced by the host against the agent during the infection. [29] Serological tests are tests such as rose Bengal tests and CFT which are facilitate the diagnosis of brucellosis in areas where 
bacterial culture not available. These serological tests include Rose Bengal test, Complement fixation tests and serum agglutination test [30]

\subsection{Rose Bengal test}

Rose Bengal test is rapid serological test of brucellosis, which is primarily designed for screening test in livestock. Positive results of rose Bengal tests are confirmed by other serological test such as Serum agglutination tests. This agglutination test is based on the reactivity of antibodies against the smooth lipopolysaccharide. Rose Bengal test has high sensitivity which is more than $99 \%$ and it had low results of false negative. Its high sensitivity makes it easy to determine the prevalence of the disease at herd level. To increase the specificity the test may be applied to a serial dilution (1:2 through 1:64) of the serum samples. [31, 32]

Although rose Bengal test has high sensitivity and which is used as screening test, it has low specificity in endemic areas and prozones. [33]

\subsection{Complement fixation test}

The Complement Fixation Test (CFT) allows the detection of anti-Brucella antibodies that are able to activate complement. Cattle immunoglobulins (Ig) that can activate bovine complement are the IgG and the IgM. According to some literature this test is not highly sensitive but shows an excellent specificity. [34, 35]

The CFT is widely used and accepted as a confirmatory test although it is complex to perform, requiring good laboratory facilities and adequately trained staff to accurately titrate and maintain the reagents. [36]

\subsection{Milk Ring Test}

It is inexpensive, easy, simple and fast to perform. It detects lacteal anti-Brucella IgM and fat globules from milk and form red ring in positive case. However, it tests false positive when milk that contains abortion in cattle. Endemic infectious causes of from cows suffering from abnormal disorder or mastitis. Milk that contain low concentration of lacteal IgM, IgA or lack the fat clustering factors, tests false negative. Because lacteal antibodies rapidly decline after abortion or parturition, the reliability of milk ring test using $1 \mathrm{ml}$ milk to detect Brucella. [37]

\subsection{Treatment}

Most of time treatments against Brucellosis are not effective because of the presence of the agent with in the cell and its ability to adapt to the environmental conditions encountered in its replicative niche e.g. macrophage, treatment of domestic animals with antibiotics is not usually successful. [38, 39, 40, 16] Although the nature of brucellosis makes it very difficult to treat, the longer treatment with antibiotics results the longer the chance of recovery. Some studies shows that the combination of antibiotics may result best way to the cure the disease. [9, 41] Even though, treatment failure and relapse rates are also common in humans, treatment depend on the drug combination of doxycycline with streptomycin which is currently the best therapeutic option with less side effects and less relapses, especially in cases of acute and localized forms of brucellosis. [42]

\section{Control And Prevention}

\subsection{Control in animals}

Though industrialized countries destroyed brucellosis from their countries, re-introduction is common risk for most countries especially for developing countries. This disease continues to exert its devastating impact perpetuating poverty. Each brucellosis control program is aimed to minimize the health and economic burden of the disease by preventing, controlling, or eliminating the disease risks originating from direct or indirect contacts with infected animals, their products, or their environment. [43]

\subsubsection{Maintaining Healthy Contact with Animals}

Since the sharing of water points for drinking and grazing lands are important risk factors for transmission of brucellosis, avoiding the mixing of cattle and other animals as well as raising replacement heifers within a herd might be important steps in the control and prevention of brucellosis in humans and animals. Screening animals before purchasing and incorporating them into a herd, the 
proper disposal of aborted materials and the isolation of animals during parturition would help to reduce the transmission of brucellosis across the herd. [43]

\subsubsection{Vaccination in Animals}

The main objective of the vaccination is to reduce release of the microorganism from infected herd as well as to reduce the incidence resulting from the disease. [44] The vaccination of the animals reduces the risk of spread of the disease to animals as well as risk of disease in humans. The most commonly used vaccines in sheep and goat is B. melitensis Rev1 (Rev1) which is available in both live and attenuated form and another widely used vaccine in cattle is B. abortus S19 (S19). Although both vaccines prompt good protection, there are some disadvantages were encountered in the vaccines. Among the disadvantages; both vaccines can cause abortion in the animals which are pregnant; it also interfere some diagnostic tests such as serology test which is required combined with test and slaughter. [12]

Although Vaccines against brucellosis have been used as a control measure, they are not 100\% effective in prevention of brucellosis. Only 65 percent of vaccinated animals are protected from becoming infected by an average exposure to Brucella Due to serious economic loss and public health risk, extensive efforts have been conducted to prevent the disease in animals through vaccination programs. [45]

In principle, vaccination against brucellosis is practiced in countries with high prevalence of more than $5 \%$, particularly in developing countries since vaccination is relatively cheap and readily acceptable by the farmers. [46]

\subsubsection{Test and Slaughter Programs}

Test-and-slaughter' is an important method to control brucellosis because it is suitable to eradicate all emerging as well as re-emerging zoonotic disease of animals. Currently most Asian countries uses Test-and-slaughter' as the control measure for brucellosis. [47] In addition to vaccination, culling of suspect or reactor animals based on serology is used in most developed countries. The crux of this strategy relies on testing of herds to determine their sero-status. In industrialized countries, animals which are positive for brucellosis are culled from the herd either by sent to slaughter or culled by government. Usually this strategy is not performed in developing countries because of financial aspect as well as lack of health infrastructure. [48]

\subsubsection{Farm Sanitation}

Farm workers and animal attendants in particular, should wear adequate protective clothing when contact with infected animals is probable or if the environment is likely to have been contaminated by excreta, abortions or parturition products from animals with brucellosis. This is particularly important when dealing with animals that are aborting or giving birth, when the shedding of Brucella organisms will reach maximum levels. Aborted fetuses, placentae and contaminated litter should be collected in leak-proof containers and disposed of preferably by incineration. Deep burial in freshly slaked lime at sites away from water courses is an acceptable alternative... [3]

\section{Public health Significance}

Brucellosis is highly infectious bacterial zoonosis which result more than 500,000 cases of human per year all around the world. [49] It is a contagious disease that human acquires the disease by direct contacts to infected animals, contact to aborted fetus, fetal membrane and discharges as well as consumption of raw dairy products from infected animal. [50] Clinical symptoms of brucellosis in human has similarity with the clinical symptom resulted from flu, that it include fever, sweats, headaches, back pains, and physical weakness. Severe infections of the central nervous systems or lining of the heart may occur. [51] Although often human is infected by the disease is through consumption of raw milk and other dairy products, infrequently human can acquire the disease through inhalation of the agent. This pathway of transmission is often seen in lab workers, veterinarians and dairy workers. [52, 53, 54]

\subsection{Control in Human}

Reservoir animals are the main source of infection in human so in order to control human brucellosis control and eradication should be done on those reservoir animals. In addition to control in reservoir 
animals, educating the society in proper handling and disposing of aborted foetus, discharges as well as associated fetal membrane can control the transmission of Brucella to human being. Educating the society not to consume row milk and other dairy products reduces the spread of disease to humans. [55]

\section{CONCLuSion}

Brucellosis is an infectious disease of animals and human. It has public health and economic importance. It is spread with in animal by ingestion of Brucella induced feed and close contact of healthy and infected animals. Human acquires the disease by consumption of raw dairy products and undercooked meat from infected animal. Developed countries had successfully eradicated the disease from their countries on test and slaughter method of control. On the contrary, this disease is still common in developing countries and severe economic losses can occur from livestock deaths, chronic disease and trade restrictions. The prevalence of Brucella can be detected by using rose Bengal test, complement fixation tests (CFT) and bacteriological tests. Therfore based on the above conclusion the following recommendations are forwarded

- Public awareness should be created in proper handling and disposing of infected birth products.

- Government should widen the availability and accessibility of effective diagnostic techniques as much as possible.

- Government should facilitate immunization of all cattles with in country

- Public awareness should be created in consumption of dairy products and meat

- Further researches and investigations should be done on the disease.

\section{REFERENCES}

[1] Pappas G. The changing Brucella ecology: novel reservoirs, new threats. International journal of antimicrobial agent 2010; 36(Suppl 1): S8-S11. [1]

[2] Ahmed, M.A.M. (2009). Seroprevalence of cattle Brucellosis in Gabiley Districts. Somaliland.58 http://www.scribd.com/doc/18682678/Abdirahim -Thesis. [2]

[3] World Health Organization 2006 Brucellosis in humans and animals [3]

[4] Wyatt HV (2005) how Hemistocles Zammit found Malta fever (brucellosis) to be transmitted by the milk of goats. J R Soc Med 98: 451-454. [4]

[5] Sriranganathan N, Seleem N, Olsen C, Samartino E, Whatmore M, et al. (2009) Brucella, In: Nene V, Kole $\mathrm{C}$ (Eds) : Genome mapping and genomics in animal-associated microbes. Springer-Verlag, Berlin, pp: 164. [5]

[6] Center for food security and public health 2018 Brucellosis: Brucella abortus [6]

[7] Malik GM. A clinical study of brucellosis in adults in the Asir region of southern Saudi Arabia. Am J Trop Med Hyg. 1997; 56:375-7. Medline:9158043 [7]

[8] PAHO-WHO (2001). Zoonoses and Communicable disease common to man, animals and wild life, Volume I. Bacterioses and Mycoses. In: Scientific and Technical publications No.580. Pan American Health Organization Regional office of the WHO, Washington D.C. USA. Pp 44-67. [8]

[9] Falagas, M.E.; Bliziotis, I.A. Quinolones for treatment of human brucellosis: Critical review of the evidence from microbiological and clinical studies. Antimicrob. Agents Chemother. 2006, 50, 22-33. [CrossRef] [PubMed] [9]

[10] Revue Scientifique et Technique (International Office of Epizootics). 2013; 32(1):271-278. [10]

[11] Nicoletti P. Brucellosis: past, present and future. Prilozi. 2010; 31(1):21-32. [11]

[12] Godfroid J, Scholz H, Barbier T, Nicolas C, Wattiau P, Fretin D, et al. Brucellosis at the animal/ecosystem/human interface at the beginning of the 21st century. Prev Vet Med. 2011;102(2):118131. pmid:21571380 View Article PubMed/NCBI Google Scholar [12]

[13] Michaux C, Bang SG, Jumas BE, Guigue T, Allardet A, O' Callaghan D, et al. Genome structure \& phylogeny in the genes Brucella. J Bacteriol. 1997; 179:3244-49. [PMC free article] [PubMed] [13]

[14] DelVecchio VG, Kapatral V, Redkar RJ, et al. The genome sequence of the facultative intracellular pathogen Brucella melitensis. Proc Natl Acad Sci USA 2002; 99: 443-8.[14]

[15] Fretin, D., Fauconnier, A., Kohler, S., Halling, S., Leonard, S., Nijskens, C., Ferooz, J., Lestrate, P., Delrue, R.M., Danese, I., Vandenhaute, J., Tibor, A., DeBolle, X., Letesson, J.J. ( 2005): The sheathed flagellum of Brucella melitensis is involved in persistence in a murine model of infection. Cell Microbiol., 7: 687-698.[15] 
[16] Seleem MN, Boyle SM, Sriranganathan N. Brucella: a pathogen without classic virulence genes. Vet Microbiol 2008; 129: 1-14.[16]

[17] Harze WD. Brucella virulence. Genome biology; 2002. p. 3. [17]

[18] Robert S. and Walter R. 2007 Large animal Theriogenology $2^{\text {nd }}$ Ed Elsevier Inc. [18]

[19] CDC Transmission of brucellosis https://www.cdc.gov/brucellosis/transmission/index.html assesed on 21/Feb/2019 [19]

[20] Arif, S.; Thomson, C.P.; Hernandez-Jover, M.; McGill, M.D.; Warriach, M.H.; Heller, J. Knowledge, attitudes and practices (KAP) relating to brucellosis in smallholder dairy farmers in two provinces in Pakistan. PLoS ONE 2017, 12, e0173365. [CrossRef] [Pub Med] [20]

[21] Currò, V.; Marineo, S.; Vicari, D.; Galuppo, L.; Galluzzo, P.; Nifosì, D.; Pugliese, M.; Migliazzo, A.; Torina, A.;Caracappa, S. The isolation of Brucella spp. from sheep and goat farms in Sicily. Small Rumin. Res. 2012, 106, S2-S5. [21]

[22] Mantur, B.G. and S.S. Mangalgi, 2007. Evaluation of conventional Castaneda and lysis centrifugation blood culture techniques for diagnosis of human brucellosis. J. clin. Microbial., 42: 4327-4328.[22]

[23] Pappas G, Papadimitriou P, Akritidis N, Christou L, Tsianos EV. The new global map of human brucellosis. Lancet Infect Dis. 2006;6:91-99. [Pub Med] [23]

[24] Ko J, Splitter GA. Molecular host-pathogen interaction in brucellosis: current understanding and future approaches to vaccine development for mice and humans. Clin Microbiol Rev. 2003; 16(1):65-78. [PMC free article] [PubMed] [24]

[25] OIE, 2000. Ovine Epididimytis (B. ovis) Manual of standard for Diagnostic Test and Vaccines. 3 ed. Rd OIE, Paris, France, pp: 467-474. [25]

[26] Al Dahouk S, Tomaso H, Nockler K, Neubauer H, Frangoulidis D. laboratory-based diagnosis of brucellosis-a review of the literature. part ii: serological tests for brucellosis. Clin lab. 2003; 49:577-89. Medline:14651329 [26]

[27] Department of agriculture, forestry and fishery republic of South Africa. BOVINE BRUCELLOSIS MANUAL 2016 [27]

[28] De Santis R, Ciammaruconi A, Faggioni G, Fillo S, Gentile B, et al. (2011) High throughput MLVA-16 typing for Brucella based on the microfluidics technology. BMC Microbiol 11: 60. [28]

[29] Juliana Pinto da Silva Mol,* Sílvia de Araújo França,* Tatiane Alves da Paixão,** Renato Lima Santos* Laboratorial diagnosis of animal brucellosis R. bras. Ci. Vet., v. 19, n. 3, p. 117-126, set./dez. 2012. [29]

[30] Ruiz JD, Sanchez G, Rehuera JM, Martin L, Lopez P, Colmenew JD. Rose Bengal test diagnosis of human bucellosis in emergency department in endemic areas. Clin Microbial Infect. 2005; 11:221-5. [PubMed] [30]

[31] Supriya Christopher, B L Umapathy, and K L RavikumarBrucellosis: Review on the Recent Trends in Pathogenicity and Laboratory Diagnosis J Lab Physicians. 2010 Jul-Dec; 2(2): 55-60. [31]

[32] Marta Pérez-Sancho, Teresa García-Seco, Lucas Domínguez and Julio Álvarez Control of Animal Brucellosis the Most Effective Tool to Prevent Human Brucellosis http://dx.doi.org/10.5772/61222 assessed on 27/02/2019 [32]

[33] Díaz R, Casanova A, Ariza J, Moriyón I (2011) He Rose Bengal Test in human brucellosis: a neglected test for the diagnosis of a neglected disease. PLoS Negl Trop Dis 5: e950. [33]

[34] Emmerzaal A, de Wit JJ, Dijkstra T, Bakker D, van Zijderveld FG. The Dutch Brucella abortus monitoring programme for cattle: the impact of false-positive serological reactions and comparison of serological tests. Vet Q. 2002; 24:40-6. [PubMed] [34]

[35] McGiven JA, Tucker JD, Perrett LL, Stack JA, Brew SD, MacMillan AP. Validation of FPA and cELISA for the detection of antibodies to Brucella abortus in cattle sera and comparison to SAT, CFT, and iELISA. J Immunol Methods. 2003; 278:171-8. doi: 10.1016/S0022-1759(03)00201-1. [PubMed] [CrossRef] [35]

[36] Xavler, M.N., T.A. Palxao, E.P. Poester, A.P. Lage and R.L. Santos, 2009. Pathology, immunohistochemistry and bacteriology of tissues and milk of cows and fetuses experimentally infected with Brucella abortus. J. Comp. Pathol., 140: 147-157.[36]

[37] Nielson, O., R. Steward, K. Nielson, L. Measurs and P. Duigan, 2001. Serologic survey of Brucella Species, antibodies in some animals of North America. J. Wild life Dis., 37: 89-100. antibodies in individual cattle or intact milk is strongly reduced [37]

[38] Berger SA. Infectious diseases of Nepal. Los Angeles: GIDEON Informatics; 2014. p. 44. [38]

[39] Plumb GE, Olsen SC, and Buttke D. Brucellosis: 'One Health' challenges and opportunities. Roushan MR, Gangi SM, Ahmadi SA. Comparison of the efficacy of two months of treatment with co-trimoxazole plus doxycycline vs. co-trimoxazole plus rifampin in brucellosis. Swiss Med Wkly. 2004; 134:564-568. [PubMed] [39] 
[40] Al-Anazi KA, Al-Jasser AM. Brucellosis: a global re-emerging zoonosis; diagnosis, treatment and prevention. 2014 [cited 2016 Oct 16]. Available from: http://www.esciencecentral.org/ebooks/bacterialmycotic-infections/brucellosis-3.php. [40]

[41] Moon, M.S. Tuberculosis of spine: Current views in diagnosis and management. Asian Spine J. 2014, 8, 97-111. [CrossRef] [Pub Med][41]

[42] Seleem, M.N., Jain, N., Pothayee, N., Ranjan, A., Riffle, J.S. and Sriranganathan, N. (2009): Targeting Brucella melitensis with polymeric nanoparticles containing streptomycin and doxycycline. FEMS Microbiol. Lett., 294: 24-31. [42]

[43] Hotez PJ, Savioli L, Fenwick A. Neglected tropical diseases of the Middle East and North Africa: review of their prevalence, distribution, and opportunities for control. PLoS Negl Trop Dis. 2012; 6(2):e1475. [43]

[44] Schuchat A, Bell BP. Monitoring the impact of vaccines post licensure: new challenges, new opportunities. Exp Rev Vacc 2008; 7(4): 437-456. [44]

[45] OIE, Terrestrial Manual. Bovine Brucellosis. Manual of Diagnostic Tests and Vaccines for Terrestrial Animals. 2009a; Chapter 2.4.3. [45]

[46] Blasco JM, Molina-Flores B. Control and eradication of Brucella melitensis infection in sheep and goats. Vet Clin North Am Food Anim Prac 2011; 27(1): 95-104. [46]

[47] Shamshad S, Zainudeen MHM, Selvarajan PR, Halim A, Abu Bakar I, Tee CH, et al. The emergence of tuberculosis in imported goats in Penang, Malaysia. In: Proceedings of the 19th Veterinary Association Malaysia Scientific Congress. Malaysia: VAM; 2007, p. 103-105. [47]

[48] Noah C. Hull and Brant A. Schumaker Comparisons of brucellosis between human and veterinary medicine. Infect Ecol Epidemiol. 2018; 8(1) [48]

[49] Seleem, M. N., Boyle, S.M. and Sriranganathan, N. (2010). Brucellosis: a re-emerging zoonosis. Veterinary Microbiology, 140(3): 392-398. [49]

[50] M.perez Ruano and MD Zambrano Aguayo study of knowledge about bovine brucellosis among people involved in cattle supply chain in the provinence of manabi, Ecuador Rev. Sci. Tech. Off. Int. Epiz., 2017, 36(3) [50]

[51] Department of Health and Human Service. Zoonotic - Brucellosis https://www.maine.gov/dhhs/ mecdc /infectious-disease/epi/zoonotic/brucellosis.shtml assessed on 05/03/2019 [51]

[52] Fact sheet brucelilosis https://www.maine.gov/dhhs/mecdc/infectious-disease/epi/documents/BrucellosisFS-2018.pdf assessed on 05/03/2019 [52]

[53] Boschiroli ML, Foulongne V, O'Callaghan D Brucellosis: a worldwide zoonosis. Curr Opin Microbiol. 2001 Feb; 4(1):58-64. [PubMed] [Ref list] [53]

[54] Mantur B.G., Biradar S.M., Bidri C.R., Mulimani S.M., Veerappa P., Kariholu P., Patil B.S., Mangalgi S.S. Protean clinical manifestations and diagnostic challenges of human brucellosis in adults: 16 years' experience in an endemic area. J. Med. Microbiol. 2006; 55:897-903. doi: 10.1099/jmm.0.46097-0. [PubMed] [CrossRef] [54]

[55] Acha PU, Szyfers B (2001): Zoonosis and Communicable Diseases Common to man and Animals. $3^{\text {rd }}$ ed. Pan America Health Organization. Washington, D.C., Pp 40-296.[55]

Citation: Tesfahun Demeke Dana, "Review on Epidemiology, Diagnosis Control and Prevention of Brucellosis" International Journal of Research Studies In Biosciences (Ijrsb), Vol. 7, no. 6, pp. 1-8, 2019. http://Dx.Doi.org/10.20431/2349-0365.0706001

Copyright: (C) 2019 Authors. This is an open-access article distributed under the terms of the Creative Commons Attribution License, which permits unrestricted use, distribution, and reproduction in any medium, provided the original author and source are credited. 